\title{
A DERMATOGLIFIA NA EDUCAÇÃO FÍSICA ESCOLAR E SUA RELAÇÃO COM O ESPORTE E A SAÚDE: UM ESTUDO DE CASO NO ESTADO DE MATO GROSSO DO SUL
}

\section{LA DERMATOGLIFIA EN LA EDUCACION FISICA ESCOLAR Y SU RELACION COM EL DEPORTE Y LA SALUD: UN ESTUDIO DE CASO EN EL DEPARTAMENTO DE MATO GROSSO DO SUL}

\author{
DERMATOGLYPHIA IN SCHOOL PHYSICAL EDUCATION AND ITS \\ RELATION TO SPORT AND HEALTH: A CASE STUDY IN MATO GROSSO DO \\ SUL'S DEPARTMENT
}

Ana Paula Moreira de SOUSA ${ }^{1}$

Deyvid Tenner de Souza RIZZO ${ }^{2}$

Vanilda Espindola da SILVA ${ }^{3}$

RESUMO: Este artigo objetiva descrever a prevalência das cristas dermopapilares de uma turma de $9^{\circ}$ ano no que se refere ao estudo da dermatoglifia, relacionando-a com a saúde e o esporte nas aulas de Educação Física escolar. A dermatoglifia é um instrumento de avaliação física e que pode ser uma proposta de aplicação no ambiente educativo. A pesquisa foi feita em uma escola pública no município de Coronel Sapucaia - Mato Grosso do Sul no ano de 2017. Foram coletadas impressões digitais de 31 estudantes, sendo 10 do sexo masculino e 21 do sexo feminino, utilizando rolo de entintamento, plaquetas, tinta para coleta de impressões digitais e folha sulfite A4. Os resultados apontaram a prevalência de presilha em ambos os sexos, portanto, os sujeitos possuem velocidade e explosão, podendo praticar o atletismo, por exemplo. É possível observar as impressões digitais dos dedos das mãos e correlacionar com potencialidades para as capacidades biofísicas, como força, velocidade, coordenação motora e resistência e aliar genótipo e fenótipo amplia as possibilidades de encaminhamento no que tange a orientação de talentos, planejamento do treinamento desportivo, preparação física e prescrição de exercícios na promoção de saúde e afins. Logo, conclui-se que o profissional de Educação Física escolar tem um papel fundamental no desenvolvimento biomotor dos estudantes, contribuindo para o aprimoramento de suas valências físicas e promoção da saúde.

PALAVRAS-CHAVE: Dermatoglifia. Educação física escolar. Esporte. Saúde.

\footnotetext{
${ }^{1}$ Faculdades Magsul (FAMAG), Ponta Porã - MS - Brasil. Docente do Curso de Educação Física. Mestre em Educação. ORCID: <https://orcid.org/0000-0003-0496-4408>. E-mail: profap.educa@gmail.com

${ }^{2}$ Universidade Federal do Maranhão (UFMA) - Campus de Pinheiro - MA - Brasil. Professor Adjunto do Curso de Educação Física no Centro de Ciências Humanas, Naturais, Saúde Tecnologia/CCHNST/. Doutor em Ciências do Desporto. ORCID: <https://orcid.org/0000-0002-9622-9816>. E-mail: deyvidrizzo1@gmail.com

${ }^{3}$ Faculdades Magsul (FAMAG), Ponta Porã - MS - Brasil. Graduada em Educação Física. ORCID: <https://orcid.org/0000-0002-4896-3944>. E-mail: vanilda_espindola@hotmail.com
} 
RESUMEN: Este artículo objetiva describir la prevalencia de las crestas dermopapilares de una clase de $9^{\circ}$ año en lo que se refiere al estudio de la dermatoglifia, relacionándola con la salud y el deporte en las clases de Educación Física escolar. La dermatoglifia es un instrumento de evaluación física y que puede ser una propuesta de aplicación en el ambiente educativo. La investigación fue realizada en una escuela pública en el municipio de Coronel Sapucaia - Mato Grosso do Sul en el año 2017. Se recolectar huellas dactilares de 31estudios, siendo 10 del sexo masculino y 21 del sexo femenino, utilizando rodillo de entintado, plaquetas, tinta para recolección de huellas dactilares y hoja sulfito A4. Los resultados apuntaron la prevalencia de presilla en ambos sexos, por lo tanto, los sujetos poseen velocidad y explosión, pudiendo practicar el atletismo, por ejemplo. Es posible observar las huellas dactilares de los dedos de las manos y correlacionar con potencialidades para las capacidades biofísicas, como fuerza, velocidad, coordinación motora y resistencia y aliar genotipo y fenotipo amplía las posibilidades de encaminamiento en lo que se refiere a la orientación de talentos, planificación del entrenamiento deportivo, preparación física y prescripción de ejercicios en la promoción de salud y afines. Por lo tanto, se concluye que el profesional de Educación Física escolar tiene un papel fundamental en el desarrollo biomotor de los estudiantes, contribuyendo para el perfeccionamiento de sus valencias físicas y promoción de la salud.

PALABRAS CLAVE: Dermatoglifia. Educación física escolar. Deporte. Salud.

ABSTRACT: This article has the objective of showing the prevalence of the dermopapillar crests of ninth year class, which is referred to as the study of Dermatoglyphic state, relating it to health and sports in PE classes at schools. Dermatoglyphia is a tool for physical evaluation and it can also be suggested to be applied in the educational environment. The analysis was done at a public school in the county of Coronel Sapucaia - Mato Grosso do Sul in the year 2017. Thirty one students had their digital prints collected, this being 10 males and 21 females, burial rolls, platelets, ink for digital print collection and A4 paper sheets were utilised for this. The results pointed out the predominance of the closeness of both sexes, so the individual has speed and explosion, therefore being able to practice sports for example. It is possible to observe the digital prints and correlate the abilities to the Biophysical capacity, such as strength, speed motor coordination and resistance, furthermore, associate genotype and phenotype, extending the routing possibilities regarding the orientation of talents. In addition, planning of the training sport, physical preparation and exercise instructions in order to encourage health promotion and what is related to it. Therefore, it is concluded that the teacher of school physical Education has a key role in the development of the biomotor ability of students, contributing to the improvement of their physical valences and health promotion.

KEYWORDS: Dermatoglyphia. School physical education. Sport. Health.

\section{Introdução}


Esta pesquisa parte da premissa de considerar seu tema na área da saúde e do esporte com sua relação no campo educacional. Trata-se, portanto de apontar o tema transversal saúde como seu ciclo inicial.

Abordar sobre o tema transversal saúde na Educação faz parte do conteúdo da Educação Física, conforme orienta os Parâmetros Curriculares Nacionais (PCN's). É citado que a criança quando inicia o ciclo escolar, traz consigo costumes e valores, sejam eles favoráveis ou não em relação à saúde, e cabe ao profissional dar continuidade no trabalho de qualidade de vida e promoção da saúde dos mesmos (BRASIL, 1997).

Esse tema é desafiante, e nesse contexto não se pretende reduzir os ensinamentos a conteúdos de higiene e saúde, mas sim apontar a necessidade da mudança de comportamentos nocivos e preservação de hábitos salutares, como por exemplo, a prática regular de esporte e exercício físico.

Ressalta-se que é importante para o estudante a prática de algum esporte, seja de rendimento ou não, para melhoria de sua qualidade de vida, aptidão física, relações sociais, etc. Nesse sentido, pontua-se que, para ter um desenvolvimento satisfatório no esporte e na saúde, por exemplo, é imprescindível considerar a dermatoglifia e o potencial genético.

Nascimento (2010) salienta que o conhecimento prévio das capacidades e tendências genéticas e sua utilização fenotípica pode contribuir para o desenvolvimento do indivíduo e determinar talentos e a dermatoglifia pode contribuir com esse processo.

Considerando, pois a dermatoglifia, ela é considerada o estudo das Impressões Digitais (ID’s) do indivíduo e é um método de observação das marcas genéticas. É um tema de fundante relevância que remete a discussões sobre saúde e esporte. De acordo com Fernandes Filho (2003, p. 158):

\footnotetext{
As impressões digitais são estudadas a mais de um século e são utilizadas como forma de diferenciação dos seres humanos. O método de análise das impressões digitais recebeu o nome de dermatoglifia e foi apresentada em 1891 por Vucetich, um iugoslavo naturalizado Argentino, que desvendou o assassinato de uma criança identificando seu assassino através das impressões digitais deixadas no sangue da vítima.
}

Entende-se que seja importante definir o sentido etimológico do termo e que segundo Fernandes Filho e Fernandes (2004), a dermatoglifia tem origem do latim e do grego, que significa "gravar na pele", tema esse proposto por Cummins e Midlo (1961). 
Ela foi introduzida na $42^{a}$ Sessão Anual da Associação Americana de Anatomos, realizada em abril de 1926.

Giraldi (2011) relata que desde o homem primitivo já havia marcas deixadas em paredes de cavernas. Essa mesma autora pontua que em países como França e Espanha são encontradas várias impressões de contornos de mãos em mais de vinte cavernas.

Contudo, observa-se que as impressões das mãos nas paredes das cavernas foram registradas a partir de diferentes traços e variações. Acreditava-se que a mão esquerda aparecia com maior frequência e a mão direita provavelmente usada nos afazeres domésticos, levando a crer que a maioria das pessoas era destra.

Ao abordar sobre dermatoglifia, é necessário considerar os sistemas de cristas e sulcos do estrato córneo da pele, que reveste a parte ventral dos dedos, das palmas das mãos e das plantas dos pés do indivíduo e isso é chamado de dermatóglifos. Além disso, tem-se figuras das cristas epidérmicas presentes nas falanges distais.

A essas cristas citadas, dar-se-á o nome de ID’s, que representam na verdade "um marcador genético, do tipo quantitativo e cujas características estatísticas configuram-se como variáveis discretas" (FERNANDES FILHO; FERNANDES, 2004, p. 158).

Considerando estas especificidades, através da dermatoglifia e leitura das cristas dermopapilares, é possível descobrir talentos no esporte, analisar a força, velocidade, resistência e coordenação motora de um indivíduo. A partir disso foi possível detectar três tipos de figuras nas digitais, quais sejam: Arco (A), Presilha (L) e Verticilo (W).

Fernandes Filho (2003, p. 90) salienta que:

As ID`s revelam em suas características, os processos de velocidade e de crescimento, e ainda permitem formar um esquema de princípios da associação das ID`s com as manifestações funcionais: resistência, velocidade, coordenação, força e as atividades cíclicas. Baseando-se nas análises das ID`s através dos tipos de desenhos: arco, presilha e verticilo, pode-se então identificar o potencial genético.

A respeito dos tipos dessas ID's, segue infracitada uma figura (Figura 1) para melhor compreensão, com o apontamento da presença de delta em algumas. 
Figura 1 - 4 tipos fundamentais de desenhos dermatoglíficos

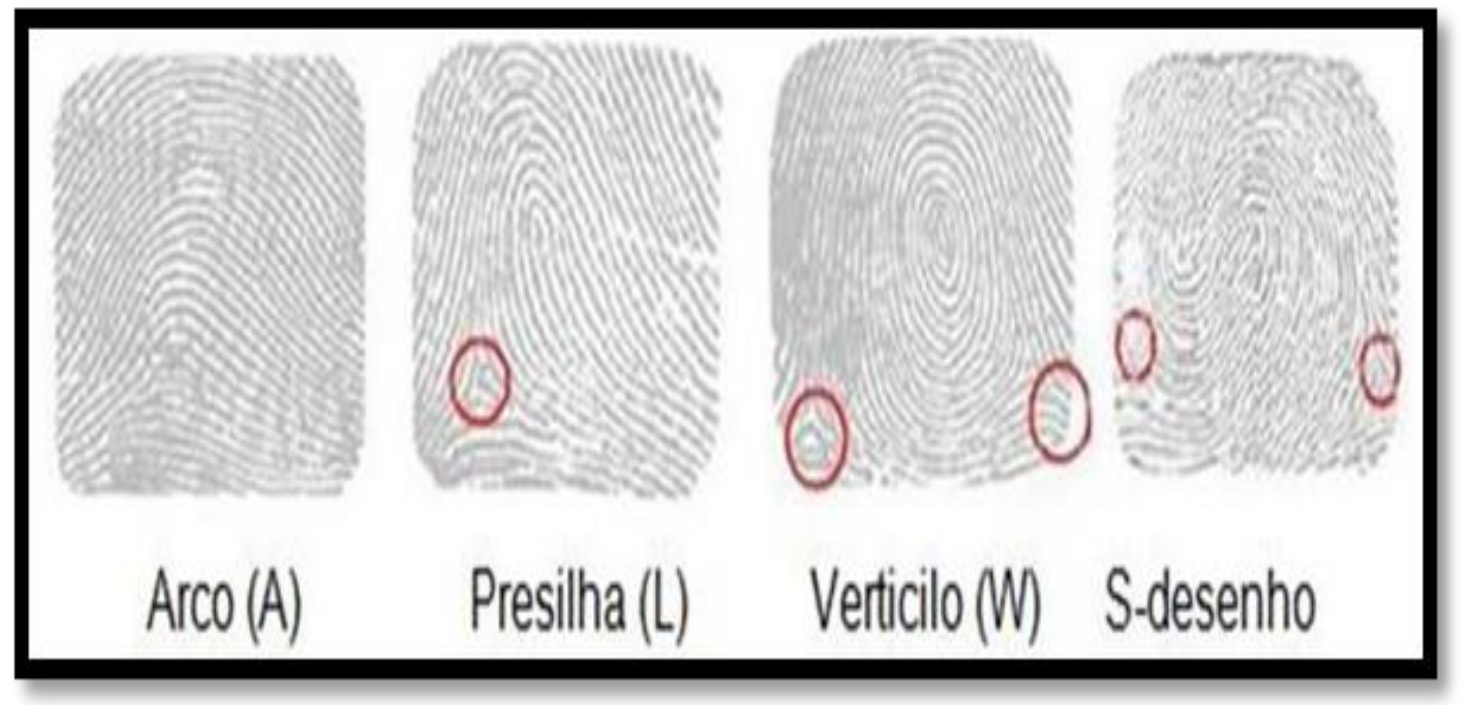

Fonte: (VAZ et al., 2011)

Giraldi (2011) afirma que as ID’s nunca são iguais em duas pessoas, mesmo que apresentem similaridades ou ainda que as diferenças possam ser marcantes e peculiares, mas que podem mesmo assim apresentar semelhança. Compreende-se, portanto que as cristas dermopapilares são semelhantes, mas nunca iguais, pois cada uma possui uma particularidade.

É preciso na verdade ser feita a coleta das ID's dos dez dedos para verificar qual será a prevalência do desenho das cristas dermopapilares do indivíduo. Um indivíduo, por exemplo, pode apresentar padrões dermatoglíifcos diferentes nos dez dedos, variando de dígito a dígito ou apresentar os mesmos padrões nos dez dedos (GIRALDI, 2011).

Levando isso em conta, é possível observar algumas informações relacionadas às capacidades físicas do indivíduo, e sobre isso, Rizzi e Marcelino (2013) pontuam que as ID's dos dedos das mãos correlacionam-se com potencialidades biofísicas, tais como força, velocidade, coordenação motora e resistência e aliar genótipo e fenótipo amplia as possibilidades de encaminhamento junto aos indivíduos no que se refere a orientação de talentos, planejamento do treinamento desportivo, preparação física e prescrição de exercícios na promoção de saúde.

Portanto, verifica-se que a dermatoglifia é um método de extrema importância para ser utilizado e trabalhado com atletas e não atletas, principalmente na orientação e planejamento/periodização de treinamento e na promoção da saúde, além de prevenção de patologias. Considera-se logo uma relação estrita entre dermatoglifia, saúde e esporte. 


\section{A relação entre saúde, esporte e dermatoglifia}

Atualmente a dermatoglifia aponta sobre esporte, pois ele é um dos seus objetos de estudo, pois como já mencionado, o estudo e a leitura de cristas papilares definem algumas características importantes para a escolha de um determinado esporte, como por exemplo.

Fernandes Filho, Dantas e Fernandes (2005, p. 152) afirmam que:

O conhecimento das características de um esporte parece propiciar a aplicação adequada das estratégias que influenciariam em seu melhor rendimento. Desta forma, vemos que as características dermatoglíficas, como marcas genéticas, bem como o somatotipo, são considerados indicativos a serem utilizados, tanto no que se refere à performance atlética, quanto na compreensão morfofuncional do atleta.

Verifica-se que no campo esportivo além do indivíduo ter prevalência das cristas dermopapilares condizentes para praticar determinado esporte, ele ainda necessita ter influências externas para que seja bem-sucedido, considerando assim seu fenótipo e genótipo.

Fernandes Filho, Dantas e Fernandes (2005) ratificam a afirmativa, ao escreverem que a predisposição genética constrói o genótipo, mas não obstante é preciso do fenótipo, isto é, as interações do indivíduo com suas práticas motoras, afetivas, sociais, etc.

Estes mesmos autores pontuam que "o modelo das impressões digitais conduz, a escolher-se mais adequadamente, a especialização no esporte com a perspectiva de otimização quanto ao talento individual" (FERNANDES FILHO; FERNANDES, 2004, p. 138).

No que se refere ao campo da saúde, Giraldi (2011) afirma que a dermatoglifia tem colaborado com o diagnóstico precoce de algumas patologias, como Síndrome de Down, Síndrome de Turner, entre outras. Ela na verdade tem seu estudo aplicado na criminalística, antropologia física, biologia humana, genética e clínica médica.

Conforme autora acima há indícios que as alterações gênicas podem mudar os dermatóglifos, e devido a isso são amplamente utilizados no diagnóstico de várias anomalias congênitas e de muitas doenças genéticas (cromossômicas e gênicas) e assim ocorrem algumas possíveis alterações dermopapilares em crianças com determinadas síndromes e doenças crônicas. 
Já foi constatado que na Síndrome de Down cerca de 35\% dos pacientes apresenta dez presilhas ulnares, encontradas somente em $4 \%$ dos indivíduos da população geral, e também um trirrádio axial característico no centro da região palmar. Na Síndrome de Klinefelter o número total de cristas é reduzido e o padrão em arco está aumentado. Na Síndrome de Turner o total de número de cristas está aumentado em relação ao indivíduo normal. A deficiência na formação das cristas é observada em $1 \%$ dos indivíduos da população geral enquanto está presente em 18\% dos casos de esquizofrenia.

Têm sido encontradas também alterações dermatoglíficas em outras doenças como o diabetes mellitus tipo 1 e 2 , doença celíaca, fenilcetonúria, hipertensão arterial, infarto do miocárdio, cardiopatia reumática, embriopatia por rubéola, leucemia, autismo, alcoolismo crônico, psoríase, alopécia areata e hanseníase (GIRALDI, 2011, p. 29).

Compreende-se que os estudos, descobertas e pesquisas feitas via dermatoglifia podem contribuir para o processo de diagnóstico precoce de doenças, auxiliando na forma de tratamento e prevenção.

Segundo Giraldi (2011), Harold Cummins e seu colaborador Charles Midlo são conhecidos como os "pais" dos dermatóglifos, pois criaram estratégias de estudo na área da dermatoglifia, que ainda são utilizadas atualmente, e afirmaram que os principais estudos foram sobre Síndrome de Down, em que a amostra apontou que existia uma prega transversal na palma da mão desses indivíduos.

Compreende-se que a dermatoglifia não trata somente da caracterização do esporte ideal, na escolha de um esporte de alto rendimento, considerando o fenótipo e genótipo do indivíduo, mas também na descoberta precoce de algumas síndromes e doenças crônicas e que isso pode ser influenciado no ambiente escolar.

\section{Caminhos metodológicos adotados}

A pesquisa caracterizou-se como pesquisa de campo, com abordagem qualiquantitativa e descritiva. O lócus investigativo foi em uma escola estadual, localizada na região urbana do município de Coronel Sapucaia - Mato Grosso do Sul (MS). Atendia 1001 estudantes, nos períodos matutino, vespertino e noturno, e inserida em um local de fácil acesso, atendendo vários bairros. A cidade é na área de fronteira, em que são atendidos estudantes residentes do país vizinho (Capitan Bado - Paraguai).

Os sujeitos da pesquisa foram estudantes do $9^{\circ}$ ano do ensino fundamental. Participaram 31, sendo 10 do sexo masculino e 21 do sexo feminino, com idade entre 14 e 15 anos. Foram utilizadas duas aulas de Educação Física para a coleta das ID's. A 
coleta aconteceu no período matutino e os estudantes foram divididos em dois grupos de acordo com o sexo.

Os instrumentos para a coleta de dados foram: rolo de entintamento, plaquetas, tinta para coleta de ID's e folha sulfite A4. A coleta foi realizada nas aulas de Educação Física, com autorização da direção e do profissional da área. O objetivo da análise das ID’s foi verificar a prevalência das cristas dermopapilares da turma selecionada.

Foi entregue a direção escolar o Termo de Compromisso para autorização da pesquisa, bem como o Termo de Consentimento e Livre Esclarecido (TCLE) aos pais ou responsáveis pelos estudantes, devidamente assinado, antes de se realizar a coleta das ID's, e além disso, os estudantes eram menores de idade e tiveram que assinar o Termo de Assentimento e Livre Esclarecido (TALE).

Após a coleta das ID's dos sujeitos, o próximo passo foi realizar as devidas análises, com caráter plausível e convincente a partir do aspecto científico e ulteriormente voltar à escola e fazer um feedback dos resultados encontrados, apontando a prevalência coletiva e individual da turma.

O protocolo utilizado foi de Cummins e Midlo (1942). Esse protocolo considera: Arco (A), que é o desenho sem deltas; presilha (L), o desenho de um delta e; verticilo (W), aquele desenho de dois deltas. Esses desenhos devem estar presentes nos dez dedos (D10). O desenho mais simples é o arco e a presilha, os intermediários são verticilo e S-desenho.

De acordo com Nascimento (2010, p. 38), as cinco combinações de desenhos são:

\begin{abstract}
AL- A presença de arco e presilha em qualquer combinação; ALW- A presença de arco, presilha e verticilo em qualquer combinação; 10Ldez presilhas; LW- a presilha e o verticilo com condição de que o numero de presilhas seja maior que cinco, ou igual a cinco; WL- O verticilo e a presilha com condição de que o número de verticilos seja maior do que cinco.
\end{abstract}

Dantas, Alonso e Fernandes Filho (2005) afirmam que há relação entre os deltas dos dez dedos e a soma total de linhas, em que o aumento de desenhos simples e a diminuição de desenhos complexos, assim como a diminuição da Somatória Total da Quantidade de Linhas (SQTL), os atletas tem uma predisposição para esportes de alta potência com um tempo curto de realização. Quando há o aumento de deltas e SQTL, o atleta possui alto nível coordenativo. Quando há valores baixos de deltas e de SQTL, são atletas com velocidade e força. 
Souza et al (2014, p. 33) salientam que:

Os desenhos são identificados através da presença ou não de delta. O arco (A) representa o desenho sem deltas ou trirrádios, sendo o mais simples e identificado pelo valor 0 , a presilha (L) é o desenho de um delta com valor 1 . Já o verticilo (W) apresenta dois deltas, sendo identificado pelo valor 2. Com a quantidade de deltas de todas as mãos, calcula-se o D10 que é o índice de trirrádios.

Portanto, o indivíduo que possui prevalência em arco tem força e pouca coordenação motora. O arco é um dos desenhos mais simples, não possui nenhum trirrádios ou delta. Logo, há um baixo desenvolvimento, ou seja, maior dificuldade dentro do esporte, jogos e brincadeiras, por apresentar força e dificuldades na coordenação motora.

Em relação à prevalência em presilha, Rolim Filho e Fernandes Filho (2007) atestam que esse indivíduo possui velocidade e explosão. É composto por um delta, é um desenho meio fechado, que inicia no extremo do dedo, encurvando, porém, mantendo uma distância da linha que se inicia.

Percebe-se que com as características dispostas, o indivíduo que possui prevalência em presilha possui velocidade e explosão, e essas especificidades podem ser desenvolvidas por meio de brincadeiras e jogos no ambiente escolar, além dos esportes que usufruem dessas valências físicas.

O verticilo é um desenho considerado importante, pois quem possui a prevalência do mesmo é considerado um indivíduo que possui boas capacidades motoras e físicas. No tocante, é um indivíduo que terá um ótimo desenvolvimento em qualquer esporte ou atividade proposta, tanto em capacidades físicas quanto motoras.

Rolim Filho e Fernandes Filho (2007), Dantas, Alonso e Fernandes Filho (2005) salientam que o indivíduo que possui prevalência nas ID's de verticilo é considerado um ser completo, tendo coordenação motora, velocidade, força, agilidade, etc. O verticilo é um dos desenhos mais complexos, pois se trata de uma figura com um desenho fechado, em que as linhas que ficam localizadas no núcleo do dedo, têm a presença de dois deltas.

Levando em consideração todas as características de cada desenho, Fernandes Filho e Fernandes (2004) apontam que a dermatoglifia poderá propiciar estratégias de treinamento, considerando as diferentes qualidades físicas envolvidas no esporte, como recurso auxiliar ao treinamento físico, e por consequência o treinamento técnico e também tático. 
Além disso, Paiva Neto e Moura (2016) afirmam que é possível através da dermatoglifia confrontar e aliar com as potencialidades do fenótipo e genótipo, e paulatinamente com a força, coordenação motora, resistência e velocidade para a escolha de um esporte e preparação física.

Acerca disso, Rizzi e Marcelino (2013, p. 108) corroboram que:

Temos que entender o funcionamento básico dos fatores que regram o corpo humano. São eles divididos em dois: o fator fenótipo, que possui influência em cerca de $30 \%$, ao passo que, o fator genótipo interfere em cerca de $70 \%$ da atividade. Essa perspectiva percentual pode ser arrastada para o cotidiano, onde ninguém tem ainda como mudar as características hereditárias, assim como cada indivíduo possui a probabilidade de herdar predisposições para desenvolvimento de algumas doenças, também possui a probabilidade de herdar habilidades físicas de seus antecedentes.

Percebe-se que o fator genótipo e carga genética são os que mais influenciam para a escolha de um esporte, para que seja realizado de forma exitosa, porém, o fator fenótipo também tem sua parcela de contribuição, pois, deve haver forças externas que levem o indivíduo a praticar o esporte.

\section{Análise dos dados}

A análise das cristas dermopapilares foi feita de maneira visual, com auxílio de uma lupa, podendo assim classificá-las em Arco, Presilha ou Verticilo. Essa análise feita teve um baixo custo e foi considerada um instrumento confiável, conforme Fernandes Filho (2003).

No processo de coleta das ID's, as falanges distais dos dez dedos foram cobertas pela por material de entitamento, em seguida eles foram colocados em uma folha sulfite A4, objetivando coletar as ID'S, tendo o devido cuidado de não borrar a impressão.

Ressalta-se que durante a pesquisa de campo houve uma troca de conhecimentos entre os pesquisadores, o profissional de Educação Física da turma que acompanhou a coleta e os sujeitos (em que mostraram - se muito interessados pelo assunto). A classificação dos desenhos das ID's foi feita através de quadro, diferenciando a prevalência de ambos os sexos, que segue infracitado. 
Quadro 1 - Resultado da coleta

\begin{tabular}{|c|c|c|c|}
\hline Classificação por sexo & Arco & Presilha & Verticilo \\
\hline Sexo feminino & 01 & 15 & 05 \\
\hline Sexo masculino & 01 & 07 & 02 \\
\hline
\end{tabular}

Fonte: Pesquisadores (2017)

O quadro 1 constituiu-se pela classificação de ambos os sexos e os desenhos das cristas dermopapilares previstas objetivaram verificar qual a prevalência das mesmas. A partir disso, observou-se que tanto o sexo feminino quanto masculino teve prevalência em presilha e menor em arco.

Como já abordado no desenvolvimento textual, o indivíduo que possui prevalência das cristas dermopapilares em presilha é aquele que possui velocidade e explosão. Dantas, Alonso e Fernandes Filho (2005, p. 107) apontam quais as modalidades a serem praticadas de acordo com a prevalência das ID's.

Modalidades com moderada a baixo nível de predisposição coordenativa estão classificadas no aparecimento do arco (A) e diminuição da parcela verticilo (W), gerando, por conseguinte a necessidade de medidas na direção de providências para solução ou mediação do problema, sugerindo, portanto acentuar as intervenções nos pontos fracos assinalados. Em esportes que necessitam de níveis acentuados de resistência de velocidade devido ao caráter anaeróbico, como é o caso das lutas, modalidades de alta performance coletiva, ex. futsal, basquetebol, handebol entre outras, apresentam perfis dermatoglíficos indicados pela presença moderada a alta de verticilo (W), moderada de presilha (L) e baixo ou ausência de arco (A), concretizando assim o aumento do D10 e SQTL.

Compreende-se que as modalidades esportivas se encaixam nas características apresentadas por cada desenho, que variam de baixa a alta intensidade e dependendo das valências físicas, o indivíduo poder ter ou não um bom desempenho.

Percebe-se que profissional de Educação Física por meio do ensino do esporte, como por exemplo, possui um papel indispensável no desenvolvimento integral dos estudantes e na conscientização para a adoção da prática esportiva mais efetiva e busca por qualidade de vida, para terem um potencial fenotípico melhorado. 


\section{Considerações finais}

Através desta pesquisa, observou-se que a dermatoglifia pode ser aplicada como recurso auxiliar pedagógico no bloco de conteúdo "conhecimento sobre o corpo" na escola, conforme previsto nos Parâmetros Curriculares Nacionais. Ela propicia ao profissional de Educação Física conhecer seus alunos, seus potenciais em relação ao esporte, suas especificidades em relação à saúde e pode ser um conteúdo complementar a ser ministrado, além daqueles já previstos pelo Referencial Curricular da Rede Estadual de Mato Grosso do Sul.

O profissional de Educação Física deve estar aberto a novos estudos, temas que tem fundante relevância para os estudantes e que enriqueça o conhecimento dos mesmos e uma aula de dermatoglifia, por exemplo, pode tornar a aula mais atrativa e interessante, eles podem se sentir mais curiosos e motivados para fazer a aula.

A partir de todas as considerações e descrições realizadas, compreende-se que a pesquisa se apontou de extrema importância para o entendimento acerca da dermatoglifia na saúde e no esporte, como sendo um método de avaliação física, que pode e deve ser utilizado no ambiente escolar.

Portanto, conclui-se que o objetivo principal elencado da pesquisa foi alcançado, isto é, descrever a prevalência das cristas dermopapilares de estudantes do $9^{\circ}$ ano, em que a partir dos resultados, constatou-se que a maioria dos sujeitos tem prevalência de desenhos nas ID's em presilha, que apresentam velocidade e explosão, podendo ser atleta de corridas (atletismo) e ginástica, como por exemplo. Sendo assim, obteve-se resultados satisfatórios, em que os sujeitos pesquisados inclusive mostraram-se interessados pela temática investigada.

\section{REFERÊNCIAS}

BRASIL. Parâmetros Curriculares Nacionais: temas transversais. Brasília: SEB/MEC: 1997.

DANTAS, P. M. S.; ALONSO, L.; FERNANDES FILHO, J. Futsal e dermatoglifia. In: DANTAS, E. H. M.; FERNANDES FILHO, J. Atividade física em ciências da saúde. Rio de Janeiro: Shape, 2005. p. 82 - 108.

FERNANDES FILHO, J. A prática da avaliação física. Shape. Rio de Janeiro, 2003.

FERNANDES FILHO, J.; FERNANDES, P. R. Estudo comparativo da dermatoglifia, somatotipia e do consumo máximo de oxigênio dos atletas da seleção brasileira de 
futebol de campo, portadores de paralisia cerebral e de atletas profissionais de futebol de campo, não portadores de paralisia cerebral. Fitness \& Performance, v. 3, n. 3, p. 158, maio/jun., 2004. Disponível em: http://fpjournal.org.br/painel/arquivos/14535_Futebol_Rev3_2004_Portugues.pdf. Acesso em: 14 set. 2017.

FERNANDES FILHO, J.; DANTAS, P. M. S.; FERNANDES, P. R. Genética e treinamento esportivo: o uso prático da dermatoglifia. Atlas do Esporte no Brasil. Rio de Janeiro: CONFEF, 2006, p. 137. Disponível em:

http://cev.org.br/arquivo/biblioteca/4013578.pdf. Acesso em: 17 set. 2017.

GIRALDI, S. Revisão histórica dos dermatóglifos e estudo comparativo entre o método tradicional de impressão palmar com tinta e método de escaneamento digital em um grupo de escolares de Curitiba, Paraná. Tese de Pós-Graduação Universidade Federal do Paraná. Curitiba, 2011. Disponível em: http://acervodigital.ufpr.br/handle/1884/29324. Acesso em: 15 out. 2017.

NASCIMENTO, J. C. Identificação dos perfis dermatoglíficos, somatotípicos e motores em atletas de futsal com idade de 13 a 15 anos do sexo masculino da Universidade de Rio Verde- Goiás. Universidade de Brasília. Programa de PósGraduação em Ciências da Saúde. Brasília- DF, 2010.

RIZZI, A. E. K.; MARCELINO, P. C. Algumas premissas sobre os estudos em dermatoglifia no esporte e nas áreas da saúde. Revista Digital, Buenos Aires, ano 18, n. 184, set. 2013. Disponível em: http://www.efdeportes.com/efd184/dermatoglifia-noesporte-e-saude.htm. Acesso em: 20 set. 2017.

ROLIM FILHO, N. G.; FERNANDES FILHO, J. Identificação do perfil dermatoglífico e somatotípico de pentatletas modernos brasileiros de alto rendimento. Revista de Educação Física, Rio de Janeiro, v. 16, n. 139, 2007. Disponível em: http://177.38.96.106/index.php/revista/article/view/503 20. Acesso em: 20 set. 2017.

SOUZA, W. C. et al. A importância da dermatoglifia na detecção de talentos no esporte: estudo de revisão. Revista Saúde e Meio Ambiente, Revista Interdisciplinar, v. 3, n. 1, p. 31-43, jan./jun. 2014. Disponível em:

http://www.periodicos.unc.br/index.php/sma/article/view/532/427. Acesso em: 18 out. 2017.

\section{Como referenciar este artigo}

SOUSA, A. P. M. de.; RIZZO, D. T. de S.; SILVA, V. E. da. A dermatoglifia na educação física escolar e sua relação com o esporte e a saúde: um estudo de caso no estado de mato grosso do sul. Temas em Educ. e Saúde, Araraquara, v. 14, n. 2, p. 229-241, jul./dez., 2018. e-ISSN 2526-3471. DOI: 10.26673/tes.v14i2.11812

Submetido em: 11/09/2018

Aprovado em: 18/10/2018 